The Astrophysical JouRnal, 504:607-611, 1998 September 10

(C) 1998. The American Astronomical Society. All rights reserved. Printed in U.S.A.

\title{
CONSTRAINTS ON THE EFFECTS OF LOCALLY BIASED GALAXY FORMATION
}

\author{
ROBERT J. SCHERRER ${ }^{1}$ \\ Department of Physics and Department of Astronomy, Ohio State University, Columbus, OH 43210 \\ AND \\ DAVID H. WEINBERG \\ Department of Astronomy, Ohio State University, Columbus, OH 43210 \\ Received 1997 December 22; accepted 1998 April 14
}

\begin{abstract}
While it is well known that "biased galaxy formation" can increase the strength of galaxy clustering, it is less clear whether straightforward biasing schemes can change the shape of the galaxy correlation function on large scales. Here we consider "local" biasing models, in which the galaxy density field $\delta_{g}$ at a point $x$ is a function of the matter-density field $\delta$ at that point: $\delta_{g}=f(\delta)$. We consider both deterministic biasing, where $f$ is simply a function, and stochastic biasing, in which the galaxy-density $\delta_{g}$ is a random variable whose distribution depends on the matter density: $\delta_{g}=X(\delta)$. We show that even when this mapping is performed on a highly nonlinear density field with a hierarchical correlation structure, the correlation function $\xi$ is simply scaled up by a constant, as long as $\xi \ll 1$. In stochastic biasing models, the galaxy autocorrelation function behaves exactly as in deterministic models, with $\bar{X}(\delta)$ (the mean value of $X$ for a given value of $\delta$ ) taking the role of the deterministic bias function. We extend our results to the power spectrum $P(k)$, showing that for sufficiently small $k$ the effect of local biasing is equivalent to the multiplication of $P(k)$ by a constant, with the addition of a constant term. If a cosmological model predicts a large-scale mass correlation function in conflict with the shape of the observed galaxy correlation function, then the model cannot be rescued by appealing to a complicated but local relation between galaxies and mass.
\end{abstract}

Subject headings: galaxies: clusters: general — galaxies: formation - large-scale structure of universe

\section{INTRODUCTION}

If galaxies form with greater efficiency (per unit mass) in high-density regions, then their clustering can be amplified with respect to that of the underlying mass distribution (Kaiser 1984). This amplification is often summarized in terms of a "bias factor" $b$, where $b^{2}=\xi_{g}(r) / \xi(r)$ is the ratio of the galaxy autocorrelation function to the mass autocorrelation function. Biased galaxy formation plays a crucial role in cosmological scenarios that assume a critical density $(\Omega=1)$ universe since these models predict excessively high velocity dispersions in galaxy groups and clusters, unless the amplitude of mass correlations is lower than the observed amplitude of galaxy correlations (Davis et al. 1985; Bardeen et al. 1986; Dekel \& Rees 1987). At first glance, it appears obvious that bias can alter the shape of the autocorrelation function in addition to changing the amplitude, since one can simply write the bias factor $b$ as a bias function $b(r)$. A physical theory of biased galaxy formation, however, cannot specify $b(r)$ directly; it can only specify how the efficiency of galaxy formation depends on environment $-b(r)$ is an output of such a theory, not an input. For example, the widely examined "high-peak" model of galaxy formation predicts a scale-independent bias factor, at least in the linear regime (Bardeen et al. 1986).

The possibility of scale-dependent bias became a serious issue once it was shown that the shape of the galaxy autocorrelation function differed from the shape predicted by the "standard" cold dark matter (CDM) model on large scales close to the linear regime (Maddox et al. 1990). With scale-dependent bias, one could, in principle, resolve this

\footnotetext{
${ }^{1}$ NASA/Fermilab Astrophysics Center, Fermi National Accelerator Laboratory.
}

discrepancy by appealing to the complex astrophysics of galaxy formation instead of altering the CDM model's fundamental cosmological assumptions (e.g., the value of $\Omega$ ). However, the specific schemes that have been proposed to achieve the requisite scale dependence are all nonlocal; the efficiency of galaxy formation is directly modulated in a coherent fashion over large scales (Babul \& White 1991; Bower et al. 1993). While this sort of coherent modulation is physically possible, it seems a priori less natural than models in which the efficiency of galaxy formation depends only on properties of the local environment. (Such nonlocal bias models also tend to break the hierarchical relation between the two-point and three-point correlation functions [Frieman \& Gaztañaga 1994].) Weinberg (1995) and Mann, Peacock, \& Heavens (1998) applied a wide range of local biasing schemes to cosmological $N$-body simulations, and they found that these schemes did not change the shape of the galaxy autocorrelation function or of its Fourier transform, the power spectrum, on large scales, though they did alter the shape in the nonlinear regime.

Is nonlocality essential to producing scale-dependent bias on large scales? In this paper we address this question analytically, extending results from earlier work. Coles (1993) showed that an arbitrary local bias applied to a Gaussian density field amplifies (or depresses) the autocorrelation function by a constant multiplicative factor. His argument works for Gaussian fields even when the rms fluctuations are nonlinear, but in the real universe the nonlinear density field cannot be Gaussian because densities cannot be negative. In practice, the efficiency of galaxy formation may depend on the mass density averaged over a fairly small, nonlinear scale, and there will almost certainly be scatter about the mean relation between galaxy and mass densities because of the influence of a variety of physical effects. 
In a seminal paper, Fry \& Gaztañaga (1993, hereafter FG) examined biasing schemes in which the galaxy density is an arbitrary function of the local mass density. FG expand the biasing function in a Taylor series and show that if the cumulants of the mass-density field exhibit hierarchical relations, then the cumulants of the locally biased galaxy-density field also exhibit hierarchical relations in the limit that $\left\langle\delta^{2}\right\rangle \ll 1$. FG examined only one-point distribution functions, but their approach can be generalized to deal with correlation functions at nonzero separation (see, e.g., Fry 1994 for a discussion of the three-point function).

The arguments in $\S \S 2$ and 3 below extend the FG results in two ways. First, we show that if the mass clustering follows a hierarchical pattern, then local bias multiplies the autocorrelation function by a constant factor on large scales (where $\xi \ll 1$ ), even if the bias is applied on a scale where the density field is nonlinear. We then show that this result carries over to stochastic local biasing, in which the galaxy density is a random variable whose mean value is a local function of the matter density. In $\S 4$ we show how our results for the galaxy autocorrelation function translate into results for the power spectrum. We summarize our conclusions in $\S 5$.

\section{DETERMINISTIC LOCAL BIAS}

A general form of deterministic local bias relates the density fluctuation field of the galaxies, $\delta_{g}$, to the density fluctuation field of the matter, $\delta$, at the same point $\boldsymbol{x}$ through an arbitrary function $f$ :

$$
\delta_{g}(\boldsymbol{x})=f[\delta(\boldsymbol{x})] .
$$

We use quantities without subscripts, such as $\delta$ and $\xi$, to refer to the underlying matter distribution, and subscripted quantities like $\delta_{g}$ and $\xi_{g}$ to refer to the biased distribution of galaxies. Although equation (1) represents the most general form of local bias in which $\delta_{g}$ is a function only of $\delta$, one could imagine more general local functions in which $\delta_{g}$ is also a function of, for example, the local velocity field or derivatives of the local gravitational potential. Implicit in equation (1) is a smoothing scale on which the continuous fields $\delta(x)$ and $\delta_{g}(x)$ are defined. Physically, this scale indicates the range over which the environment directly influences the efficiency of galaxy formation. In a random field with significant long-wavelength power, the local density contrast is itself correlated with the density contrast on larger scales, and it is this correlation that allows a local transformation to amplify $\xi(r)$ by a constant factor on large scales (Kaiser 1984); however, the Bower et al. (1993) model for scale-dependent bias effectively incorporates an "influence" scale of tens of Mpc, which implies that a forming galaxy is "aware" of the physical conditions far away. We do not consider here the time evolution of the bias (see Fry 1996 and Tegmark \& Peebles 1998 for such a discussion); our bias function represents the present-day relation between $\delta$ and $\delta_{g}$.

Coles (1993) and FG have demonstrated a number of important properties of biasing models defined by equation (1). For the case where $\delta$ is a Gaussian field, Coles (1993) shows that $\xi_{g}(r) \propto \xi(r)$ on all scales where $\xi(r) \ll 1$ for almost any choice of the function $f$. In other words, arbitrary local biasing of a Gaussian density field does not alter the shape of the autocorrelation function on large scales. Coles also notes that his argument fails for some simple, albeit physically unlikely, functions, such as $\delta_{g}=\delta^{2}-\left\langle\delta^{2}\right\rangle$.
FG expand the function $f$ in a Taylor series:

$$
f(\delta)=\sum_{k=0}^{\infty} \frac{b_{k}}{k !} \delta^{k}
$$

where $b_{0}$ is chosen to give $\left\langle\delta_{g}\right\rangle=0$. They then derive the cumulants of $\delta_{g}$ in terms of the cumulants of $\delta$ and the biasing coefficients $b_{k}$, in the limit that $\left\langle\delta^{2}\right\rangle \ll 1$. In this limit, it is obvious from equation (2) that the leading-order effect on the variance is $\sigma_{g}^{2}=b_{1}^{2} \sigma^{2}$; in FG's notation

$$
\bar{\xi}_{g, 2}=b_{1}^{2} \bar{\xi}_{2}+O\left(\bar{\xi}_{2}^{2}\right),
$$

where $\overline{\xi_{2}}=\left\langle\delta^{2}\right\rangle=\sigma^{2}$ (see FG, eq. [9]).

With the FG expansion (eq. [2]), the galaxy autocorrelation function can be written

$$
\begin{aligned}
\xi_{g}\left(x_{1}, x_{2}\right) & =\left\langle\delta_{g}\left(x_{1}\right) \delta_{g}\left(x_{2}\right)\right\rangle, \\
& =\sum_{j, k=0}^{\infty} \frac{b_{j} b_{k}}{j ! k !}\left\langle\delta(x)^{j} \delta\left(x_{2}\right)^{k}\right\rangle .
\end{aligned}
$$

If the smoothing scale on which $\delta(x)$ is defined is large enough, then $\left\langle\delta^{2}\right\rangle \ll 1$, and only the $j=k=1$ term survives, implying that (Gaztañaga \& Baugh 1998)

$$
\xi_{g}\left(x_{1}, x_{2}\right)=b_{1}^{2} \xi\left(x_{1}, x_{2}\right)+O\left(\xi^{2}\right) .
$$

In other words, if there is a deterministic local relation between galaxy density and mass density on some scale in the linear regime, then the autocorrelation function in the linear regime is multiplied by a scale-independent factor $b_{1}^{2}$, where $b_{1}$ is the first derivative of the local bias function $f(\delta)$ evaluated at $\delta=0$. This argument is a trivial extension of the one-point argument for the variance given by FG, analogous to Fry's (1994) extension of the FG skewness result to the three-point correlation function.

What if the density field is nonlinear on the scale where local bias operates, so that $\left\langle\delta^{2}\right\rangle \gtrsim 1$ ? This situation is physically plausible, and we are no longer free to discard the higher order terms in the sum in equation (5). We can still make progress if we introduce the assumption that the clustering is hierarchical, i.e., the connected part of $\left\langle\delta\left(x_{1}\right)^{j} \delta\left(x_{2}\right)^{k}\right\rangle$ is given by (Peebles 1980; Fry 1984; Bernardeau 1996)

$$
\left\langle\delta\left(x_{1}\right)^{j} \delta\left(x_{2}\right)^{k}\right\rangle_{c}=C_{j, k}\left\langle\delta^{2}\right\rangle^{j+k-2}\left\langle\delta\left(x_{1}\right) \delta\left(x_{2}\right)\right\rangle+O\left(\xi^{2}\right) .
$$

Although the assumption of hierarchical clustering can be shown to be rigorously valid only in the quasi-linear regime, numerical simulations show that it holds to a fairly good approximation even in the nonlinear regime (Colombi, Bouchet, \& Schaeffer 1994; Colombi, Bouchet, \& Hernquist 1996; see Suto \& Matsubara 1994 for the opposing point of view), and there are theoretical grounds for believing that hierarchical clustering should apply in the nonlinear regime (Davis \& Peebles 1977; Peebles 1980; Balian \& Schaeffer 1989). There is also support for hierarchical clustering in the observed galaxy distribution (see, for example, Szapudi et al. 1995), but this is not directly relevant to our argument since we are interested in the dark matter clustering hierarchy, which cannot be observed directly. The validity of equation (7) for our evolved density field is the key assumption we make in this section; it allows us to generalize equation (6) to the biasing of nonlinear fields. Bernardeau (1996) begins with equation (7) and derives a gravitationally induced "bias," but this differs from the arbitrary bias functions we are dealing with here. 
With the hierarchical assumption, we can write

$$
\begin{aligned}
\xi_{g}\left(x_{1}, x_{2}\right)= & \sum_{j, k=0}^{\infty} \frac{b_{j} b_{k}}{j ! k !}\left[C_{j, k}\left(\sigma^{2}\right)^{j+k-2} \xi\left(x_{1}, x_{2}\right)\right. \\
& \left.+O\left(\xi^{2}\right)+\left\langle\delta\left(x_{1}\right)^{j} \delta\left(x_{2}\right)^{k}\right\rangle_{\text {unconnected }}\right] .
\end{aligned}
$$

The first two terms arise from the connected part of $\left\langle\delta\left(x_{1}\right)^{j} \delta\left(x_{2}\right)^{k}\right\rangle$, while the last term is the unconnected part. Note, however, that this unconnected part can be written as powers of lower order correlations, which can themselves be expanded out according to equation (7). [There are no terms of zeroth order in $\xi$ arising from the unconnected terms of the form $\left\langle\delta\left(x_{1}\right)^{j}\right\rangle\left\langle\delta\left(x_{2}\right)^{k}\right\rangle$, because all such terms are cancelled by other terms included in $b_{0}$.] In the end, we obtain

$$
\xi_{g}\left(x_{1}, x_{2}\right)=\left[\sum_{j k} K_{j, k} \frac{b_{j} b_{k}}{j ! k !}\left(\sigma^{2}\right)^{j+k-2}\right] \xi\left(x_{1}, x_{2}\right)+O\left(\xi^{2}\right),
$$

where $K_{j, k}$ is a set of constants. Hence, we find that for $\xi \ll 1$, the quantity $b^{2}=\xi_{g}(r) / \xi(r)$ is approximately constant. Again, we wish to emphasize that we have assumed nothing about the linearity of the density field at the scale of biasing; all we have assumed is the validity of equation (7).

Both the Coles (1993) result for Gaussian initial conditions and the FG result (eq. [6]) are special cases of our general result. If the underlying mass-density field is Gaussian, as in the case discussed by Coles (1993), then the density field is hierarchical in the sense that equation (7) is satisfied, but all of the hierarchical coefficients $C_{j, k}$ vanish except for $C_{1,1}$, which is unity. Then our conditions are satisfied, and $\xi_{g}\left(x_{1}, x_{2}\right)=b^{2} \xi\left(x_{1}, x_{2}\right)$. Formally, the Coles result holds even for the case $\left\langle\delta^{2}\right\rangle>1$, but this is not a physically realistic case, since the density field will be Gaussian only for $\left\langle\delta^{2}\right\rangle \ll 1$.

To obtain the FG result, we simply take $\sigma^{2} \ll 1$ in equation (8). Then the $j=k=1$ term dominates, and we reobtain equation (6). This equation differs from our more general result in that if the local bias is applied on a nonlinear scale, then all of the Taylor series coefficients of the bias function contribute to determining the bias factor on large scales, not just $b_{1}$.

Our argument for scale-independent bias fails when $\xi$ becomes larger than unity, which is a good thing, since local bias can change the shape of the autocorrelation function and power spectrum in this regime (Weinberg 1995; Mann et al. 1998). Note, however, that our argument does hold even for the case of quadratic biasing, $f(\delta)=\delta^{2}-\left\langle\delta^{2}\right\rangle$. The reason that the Coles (1993) argument fails in this case is that a Gaussian density field has no connected higher moments, so terms linear in $\xi$ vanish.

\section{STOCHASTIC LOCAL BIAS}

The bias model of equation (1) can at best be an idealization. Even in the case where galaxies "trace the mass," $f(\delta)=\delta$, there will be Poisson fluctuations about the mean relation because of the discrete nature of the galaxy distribution. More generally, we expect the probability of forming a galaxy in a given region to depend on many factors, including the history of accretion and mergers in the nearby environment. Many of these factors will be correlated with the local density, but they will not be completely determined by it. We can quantify our ignorance by allow- ing for stochastic bias, in which the galaxy density is a random variable which depends in some way on the underlying matter density, but which is not completely determined by it. Little previous work has been done on stochastic bias models, although Pen (1997) has recently attempted to model the joint galaxy-matter probability distribution function using a bivariate Gaussian as a starting point. Other recent discussions of stochastic biasing include those of Dekel (1997), Dekel \& Lahau (1998), and Tegmark \& Peebles (1998).

Let us therefore assume that the galaxy-density $\delta_{g}$ at a point $x$ is a random variable $X$, which is a function of the underlying matter density at that same point:

$$
\delta_{g}(x)=X[\delta(x)] .
$$

We again assume that $X$ includes a constant term that gives $\left\langle\delta_{g}\right\rangle=0$. As in the case of deterministic local bias, we assume some smoothing scale over in which $\delta_{g}$ and $\delta$ are defined, so that the bias does not occur at a geometric point but over some small volume. The random variable $X$ is uniquely specified by the probability of producing a particular value of $X$, given an underlying value of $\delta$, which we write in the standard way as $p(X \mid \delta)$, the probability of $X$ given $\delta$. Note that our assumption that the stochastic bias is purely local is actually very restrictive. It means, for example, that the distribution of the random variable $X$ is the same at every point in space with the same $\delta$, and that there are no correlations between this distribution at different points in space.

The probability of measuring a galaxy-density $\delta_{g 1}$ at the point $x_{1}$, and a galaxy-density $\delta_{g 2}$ at the point $x_{2}$ is

$$
P\left(\delta_{g 1}, \delta_{g 2}\right)=\int P\left(X_{1} \mid \delta_{1}\right) P\left(X_{2} \mid \delta_{2}\right) p\left(\delta_{1}, \delta_{2}\right) d \delta_{1} d \delta_{2},
$$

where we have used subscripts 1 and 2 to denote the values of $X$ and $\delta$ at the points $x_{1}$ and $x_{2}$, and $p\left(\delta_{1}, \delta_{2}\right)$ to denote the two-point probability distribution of the matter density at these points. (Note that $\delta_{g}=X$ in this equation.) For this model, the galaxy autocorrelation function is

$$
\begin{aligned}
\xi_{g}\left(x_{1}, x_{2}\right)= & \left\langle X\left(x_{1}\right) X\left(x_{2}\right)\right\rangle, \\
= & \int X_{1} X_{2} P\left(X_{1} \mid \delta_{1}\right) P\left(X_{2} \mid \delta_{2}\right) p\left(\delta_{1}, \delta_{2}\right) \\
& \times d \delta_{1} d \delta_{2} d X_{1} d X_{2} .
\end{aligned}
$$

We can perform the integration over $X_{1}$ and $X_{2}$ to obtain

$$
\xi_{g}\left(x_{1}, x_{2}\right)=\int \bar{X}\left(\delta_{1}\right) \bar{X}\left(\delta_{2}\right) p\left(\delta_{1}, \delta_{2}\right) d \delta_{1} d \delta_{2},
$$

where $\bar{X}(\delta)$ is the mean value of $X$ for a given value of $\delta$. This result generalizes in a straightforward way to all of the higher-order correlation functions.

The argument that leads from equation (10) to equation (14) is almost trivial, but the result is rather remarkable. It shows that the calculation of the correlation function for the most general possible stochastic local biasing model can be reduced to the equivalent problem for a deterministic local bias, with $\bar{X}(\delta)$ taking the role of the bias function. Hence, all of the mathematical machinery developed here and in other papers for the problem of deterministic local bias can be used for stochastic bias. Thus far, we have made no assumptions about the underlying density field $\delta$. If we now repeat our assumption from the previous section that $\delta$ exhibits hierarchical clustering, then we obtain the same 
result as in the previous section: $\xi_{g} / \xi$ is constant as long as $\xi \ll 1$. Equation (14) takes a particularly simple form if galaxies trace the mass on average, $\bar{X}(\delta)=\delta$. In this case, we obtain simply $\xi_{g}(r)=\xi(r)$. This result tells us that the random fluctuations about the mean density make no difference in the final autocorrelation function.

These results may seem counterintuitive, since stochastic bias ought to introduce some sort of increased "scatter" in the final density distribution, and it certainly increases the final rms density fluctuation. One must remember, however, that $\xi_{g}$ represents a volume-averaged correlation function, within which all of the random fluctuations have been averaged out. What does change for the case of stochastic bias are the random fluctuations relative to $\xi_{g}$. The variance of the autocorrelation function at some fixed separation is

$$
\begin{aligned}
\sigma_{\xi}^{2}= & \int\left(\delta_{g 1} \delta_{g 2}\right)^{2} p\left(\delta_{g 1}, \delta_{g 2}\right) d \delta_{g 1} d \delta_{g 2} \\
& -\left[\int \delta_{g 1} \delta_{g 2} p\left(\delta_{g 1}, \delta_{g 2}\right) d \delta_{g 1} d \delta_{g 2}\right]^{2} .
\end{aligned}
$$

In terms of our stochastic bias function $X(\delta)$, this becomes

$$
\begin{aligned}
\sigma_{\xi}^{2}= & \int \overline{X_{1}\left(\delta_{1}\right)^{2} X_{2}\left(\delta_{2}\right)^{2}} p\left(\delta_{1}, \delta_{2}\right) d \delta_{1} d \delta_{2} \\
& -\left[\int \bar{X}_{1}\left(\delta_{1}\right) \bar{X}_{2}\left(\delta_{2}\right) p\left(\delta_{1}, \delta_{2}\right) d \delta_{1} d \delta_{2}\right]^{2} .
\end{aligned}
$$

To illustrate the way in which $\sigma_{\xi}^{2}$ is increased, we consider again the simple class of models in which $\bar{X}(\delta)=\delta$, and we use equation (16) to calculate the difference between $\sigma_{\xi}^{2}$ for the stochastic case, and $\sigma_{\xi}^{2}$ for the deterministic case $\delta_{g} \stackrel{\xi}{=} \delta$ :

$\sigma_{\xi}^{2}$ (stochastic) $-\sigma_{\xi}^{2}$ (deterministic)

$$
=\int \sigma_{X}^{2}\left(\delta_{1}\right) \sigma_{X}^{2}\left(\delta_{2}\right) p\left(\delta_{1}, \delta_{2}\right) d \delta_{1} d \delta_{2} .
$$

Here $\sigma_{X}^{2}(\delta)$ is the variance of the distribution of $X$ for a given value of $\delta$,

$$
\sigma_{X}^{2}(\delta)=\overline{X(\delta)^{2}}-\bar{X}(\delta)^{2} .
$$

Since $\sigma_{X}^{2}(\delta)$ is positive, this result shows that randomness in the bias function increases the fluctuations about the mean value of $\xi_{g}$. Conceptually speaking, equations (15)-(17) presume that one estimates $\xi_{g}(r)$ from many different pairs of positions with spatial separation $r$ (or from a single pair of positions in an ensemble of universes) and computes the variance $\sigma_{\xi}^{2}$ of these estimates. In practice, one must average over a large number of position-pairs in order to get an estimate of $\xi_{g}$ that is at all useful, but stochastic biasing will still act to increase the variance in estimates of $\xi_{g}$ from one volume of the universe to another. These fluctuations, which can be measured in large redshift surveys, encode information about the degree of stochasticity in the galaxy formation process at fixed local mass density. (Of course, even in the absence of stochastic bias, the variance in $\xi_{g}$ is nonzero.)

The rms fluctuation of a smoothed field can be written as an integral over $\xi(r)$. The conclusion that $\xi_{g}(r)=\xi(r)$ for $\bar{X}(\delta)=\delta$ at first seems to contradict the obvious fact that stochasticity will increase the rms fluctuations smoothed on any length scale; however, these two results are not contradictory. Recall that we assumed the initial density field is smoothed over some scale $R_{s}$ and that local bias operates over this same smoothing scale. Our assumption that the distribution of $X$ is uncorrelated at different points is invalid for separations less than $R_{s}$, which means equation (11) also fails on such short separations. This is most obvious for the case of zero separation. If we measure the density at a single point $x_{1}$, then the product of probabilities $P\left(X_{1} \mid \delta_{1}\right) P\left(X_{2} \mid \delta_{2}\right)$ in equation (12) must be replaced by the single probability $P\left(X_{1} \mid \delta_{1}\right)$, and equation (12) becomes

$$
\begin{aligned}
\xi_{g}\left(x_{1}, x_{2}\right) & =\left\langle X\left(x_{1}\right) X\left(x_{2}\right)\right\rangle, \\
& =\int X_{1} X_{1} P\left(X_{1} \mid \delta_{1}\right) p\left(\delta_{1}\right) d \delta_{1} d X_{1} .
\end{aligned}
$$

Integrating over $X_{1}$ gives

$$
\xi_{g}\left(x_{1}, x_{2}\right)=\int \overline{X(\delta)^{2}} p(\delta) d \delta .
$$

For the deterministic case where $\delta_{g}=\delta$, the corresponding quantity is

$$
\xi_{g}\left(x_{1}, x_{2}\right)=\int \delta^{2} p(\delta) d \delta
$$

For the special case where $\bar{X}(\delta)=\delta$, we have

$\xi_{g}$ (stochastic) $-\xi_{g}$ (deterministic)

$$
=\int\left[\overline{X(\delta)^{2}}-\bar{X}(\delta)^{2}\right] p(\delta) d \delta .
$$

But $\overline{X(\delta)^{2}}-\bar{X}(\delta)^{2}>0$ for all values of $\delta$, so $\xi_{g}$ (stochastic) $-\xi_{g}$ (deterministic) $>0$. Thus, stochastic bias increases the rms fluctuations, but the entire effect is due to the change in $\xi_{g}(r)$ at separations smaller than our initial smoothing length; at these length scales our arguments regarding the effects of stochastic bias on the autocorrelation function do not apply.

\section{THE POWER SPECTRUM}

Although we have focused so far on the autocorrelation function, many observational studies of large-scale structure use its Fourier transform, the power spectrum, to quantify clustering on the largest scales. The Mann et al. (1997) numerical study of local biasing focuses mainly on the power spectrum. The mass power spectrum $P(k)$ is related to the mass autocorrelation function $\xi(r)$ by

$$
P(k)=4 \pi \int \xi(r) \frac{\sin (k r)}{k r} r^{2} d r,
$$

and the galaxy power spectrum is

$$
P_{g}(k)=4 \pi \int \xi_{g}(r) \frac{\sin (k r)}{k r} r^{2} d r .
$$

In $\S 2$, we showed that deterministic local bias applied to a hierarchically clustered density field gives $\xi_{g}(r)=b^{2} \xi(r)$ for $\xi(r) \ll 1$, but we can put no constraint on the bias for $\xi(r) \gtrsim$ 1 . Let $r_{0}$ be a distance such that $\xi(r) \ll 1$ when $r>r_{0}$. We can therefore write

$$
\xi_{g}(r)=b^{2} \xi(r)+\tilde{\xi}(r),
$$

where $\tilde{\xi}(r)=0$ for $r>r_{0}$. Substituting this equation into equations (24) and (25), we get

$$
P_{g}(k)=b^{2} P(k)+4 \pi \int_{0}^{r_{0}} \tilde{\xi}(r) \frac{\sin (k r)}{k r} r^{2} d r .
$$

If we choose a fixed value of $k$ for which $k r_{0} \ll 1$, then $k r \ll 1$ over the entire range of integration in the second 
term, so this integral just reduces to $4 \pi \int_{0}^{r_{0}} \tilde{\xi}(r) r^{2} d r$, which is a constant, independent of $k$. Thus, in the regime $k \ll 1 / r_{0}$,

$$
P_{g}(k)=b^{2} P(k)+c,
$$

where $b$ is the large-scale bias factor of the autocorrelation function and $c$ is a constant, which may be positive or negative. This is just a more rigorous way of noting that the power spectrum for $k<k_{0}$ is dominated by the correlation function at $r>1 / k_{0}$, though small scale fluctuations can add a constant offset to $P(k)$. Equation (28) is not quite the same as a scale-independent amplification of $P(k)$. The power spectrum estimated by Baugh \& Efstathiou (1993) from the APM survey, however, continues to rise out to $2 \pi / k \gtrsim 130 h^{-1} \mathrm{Mpc}$, so in realistic models the constant $c$ is likely to become unimportant on large scales, at least until one reaches the turnover in the power spectrum.

\section{CONCLUSIONS}

We have shown that for a local bias function applied to a density field with a hierarchical correlation structure, the only effect is to rescale the autocorrelation function by an overall bias factor $b^{2}$ on length scales for which $\xi(r) \ll 1$; no change in the shape of the autocorrelation function can be induced by such a local transformation. For the power spectrum, for sufficiently small $k$, the result is also a rescaling, with the possible addition of a constant term. Although we have assumed hierarchical clustering, our result holds as long as

$$
\left\langle\delta\left(x_{1}\right)^{j} \delta\left(x_{2}\right)^{k}\right\rangle=D_{j, k}\left\langle\delta\left(x_{1}\right) \delta\left(x_{2}\right)\right\rangle+O\left(\xi^{2}\right),
$$

where $D_{j, k}$ is independent of the separation between $x_{1}$ and $\boldsymbol{x}_{2}$. Equation (29) is actually a slightly weaker condition than the assumption of hierarchical clustering (eq. [7]), because the moment on the left-hand side of equation (29) is not connected.

If there is a bias between galaxies and mass (and the galaxy morphology-density relation implies that there must be bias for at least some kinds of galaxies), then the physics that causes it may well be complex. Our stochastic biasing result, however, implies that all environmental effects on the efficiency of galaxy formation influence $\xi_{g}(r)$ only to the extent that they are correlated with the mass density itself, and if these effects are local, then they still will not change the shape of the autocorrelation function on scales in the linear regime. Cen \& Ostriker (1992, Fig. 4) presented a first attempt to calculate the full distribution function $P\left(\delta_{g} \mid \delta\right)$ using a hydrodynamic cosmological simulation of the standard CDM model. We can expect substantial progress from this a priori approach to biased galaxy formation over the next few years, since advances in computer power and algorithms now allow simulations of much higher dynamic range and permit broader explorations of cosmological parameter space. Our results imply, however, that all of these calculations should produce galaxy populations with $\xi_{g}(r) \propto \xi(r)$ on large scales. Only a biasing mechanism that coherently modulates galaxy luminosities on scales larger than those over which the matter actually moves, e.g., suppression or enhancement of star formation by quasar radiation (Babul \& White 1991; Bower et al. 1993), can rescue a cosmological model that predicts the wrong shape for $\xi(r)$ on the scales where $\xi(r) \ll 1$. Since a physical mechanism of this sort would surely have a different impact on galaxies of different luminosities and morphological types, the giant redshift surveys becoming available in the next few years will allow us to test whether nonlocal biasing occurred in the real universe by comparing the large-scale correlation functions of different classes of galaxies.

R. J. S. and D. H. W. were supported in part by the NASA Astrophysical Theory Program through grant NAG 5-3111 at Ohio State University. R. J. S. was supported in part by NASA grant NAG 5-2788 at Fermilab and by DOE grant DE-FG02-91ER40690 at Fermilab and Ohio State University.
Babul, A., \& White, S. D. M. 1991, MNRAS, 253, L31

Balian, R., \& Schaeffer, R. 1989, A\&A, 226,373

Bardeen, J., Bond, J. R., Kaiser, N., \& Szalay, A. 1986, ApJ, 304, 15

Baugh, C. M., \& Efstathiou, G. 1993, MNRAS, 265, 145

Bernardeau, F. 1996, A\&A, 312, 11

Bower, R. G., Coles, P., Frenk, C. S., \& White, S. D. M. 1993, ApJ, 405, 403

Cen, R., \& Ostriker, J. P. 1992, ApJ, L113, 1992

Coles, P. 1993, MNRAS, 262, 1065

Colombi, S., Bouchet, F. R., \& Hernquist, L. 1996, ApJ, 465, 14

Colombi, S., Bouchet, F. R., \& Schaeffer, R. 1994, A\&A, 281, 301

Davis, M., Efstathiou, G., Frenk, C. S., \& White, S. D. M. 1985, ApJ, 292, 371

Davis, M., \& Peebles, P. J. E. 1977, ApJS, 34, 425

Dekel, A. 1997, in Galaxy Scaling Relations: Origins, Evolution, and

Applications, ed. L. da Costa \& A. Renzini (Berlin: Springer), 245

Dekel, A., \& Lahau, O. 1998, ApJ, submitted

Dekel, A., \& Rees, M. J. 1987, Nature, 326, 455

Frieman, J. A., \& Gaztañaga, E. 1994, ApJ, 425, 392

Fry, J. N. 1984, ApJ, 279, 499

\section{REFERENCES}

Fry, J. N. 1994, Phys. Rev. Lett., 73, 215

Fry, J. N. 1996, ApJ, 461, L65

Fry, J. N., \& Gaztañaga, E. 1993, ApJ, 413, 447

Gaztañaga, E., \& Baugh, C. M. 1998, MNRAS, 294, 229

Kaiser, N. 1984, ApJ, 284, L9

Maddox, S. J., Efstathiou, G., Sutherland, W. J., \& Loveday, J. 1990, MNRAS, 242, L43

Mann, R. G., Peacock, J. A., \& Heavens, A. F. 1998, MNRAS, 293, 209 (astro-ph/9707018)

Peebles, P. J. E. 1980, The Large-Scale Structure of the Universe (Princeton: Princeton Univ. Press)

Pen, U.-L. 1997, ApJ, in press

Suto, Y., \& Matsubara, T. 1994, ApJ, 420, 504

Szapudi, I., Dalton, G., Efstathiou, G. P., \& Szalay, A. 1995, ApJ, 444, 520

Tegmark, M., \& Peebles, P. J. E. 1998, ApJ, in press

Weinberg, D. H. 1995, in Wide-Field Spectroscopy and the Distant Universe, ed. S. J. Maddox \& A. Aragón-Salamanca (Singapore: World Scientific), 129 